\section{Towards Protein Field-Effect Transistors: Report and Model of a Prototype $^{* * *}$}

\author{
By Giuseppe Maruccio, ${ }^{*}$ Adriana Biasco, \\ Paolo Visconti, Alessandro Bramanti, \\ Pier Paolo Pompa, Franco Calabi, Roberto Cingolani, \\ Ross Rinaldi, Stefano Corni, Rosa Di Felice, \\ Elisa Molinari, Martin P. Verbeet, and \\ Gerard W. Canters
}

Since the early 1970s, the electronics industry has been essentially identified with metal-oxide semiconductor (MOS) large-scale integrated circuits. During the past decades, remarkable advances have been accomplished by the downsizing of components (such as MOS field-effect transistors), and the number of transistors on a chip has continuously increased in accordance with Moore's law thanks to constant improvements in lithographic resolution (the top-down approach). However, this approach is unlikely to be sustainable due to intrinsic physical limitations and to the vast increase in production costs. Molecular electronics was proposed in 1974 by Aviram and Ratner ${ }^{[1]}$ as an alternative bottom-up approach for either standard devices (such as diodes and transistors) or new functional devices. It aims to exploit the unique features of molecular systems, such as the high reproducibility and small size of the building blocks, thermodynamically driven self-assembly, and self-recognition. Today, the obstacles to the development of molecular electronics devices appear more technical than conceptual ${ }^{[2-4]}$ the main problems are the development of reliable methods to interconnect molecules, to characterize and understand their electronic properties, and to exploit them in real devices.

In this work, we take advantage of the redox properties and the functional groups of a protein, blue-copper azurin, to achieve a hybrid transistor based on proteins covalently bonded in ordered layers onto $\mathrm{Si} / \mathrm{SiO}_{2}$ substrates. This is a different and innovative approach with respect to those based on

[*] Dr. G. Maruccio, Dr. A. Biasco, Dr. P. Visconti, Dr. A. Bramanti, Dr. P. P. Pompa, Dr. F. Calabi, Prof. R. Cingolani, Prof. R. Rinaldi National Nanotechnology Laboratory of INFM, University of Lecce Via per Arnesano, I-73100 Lecce (Italy)

E-mail: giuseppe.maruccio@unile.it

Dr. G. Maruccio

Department of Physics, University of Lecce

Via per Arnesano, I-73100 Lecce (Italy)

Dr. S. Corni, Dr. R. Di Felice, Prof. E. Molinari

INFM Center on nanoStructures and bioSystems at Surfaces S3 Via Campi 213/A, I-41100 Modena (Italy)

Dr. M. P. Verbeet, Prof. G. W. Canters

Gorlaeus Laboratoria, Leiden University

Einsteinweg 55, NL-2333 CC Leiden (The Netherlands)

[**: This work was supported in part by the EU (SAMBA Project) and by the Italian MIUR (FIRB Project "Molecular Devices"). We thank Eliana D'Amone for her technical help. physisorbed monolayers obtained by evaporation or spincoating, or based on single nanosized objects like carbon nanotubes that have serious interconnection problems. ${ }^{[5]}$ The integrity of proteins in dry monolayers is investigated by intrinsic fluorescence spectroscopy, and a model for transport due to the novelty of the material is also proposed.

Azurin from P. aeruginosa (Fig. 1b, inset) is a $14.6 \mathrm{kDa}$ blue-copper protein that, in vitro, is able to mediate electron transfer (ET) from cytochrome $\mathrm{c}_{551}$ to nitrite reductase from the same organism. ${ }^{[6]}$ Azurin exists in two stable configurations $-\mathrm{Cu}^{\mathrm{I}}$ and $\mathrm{Cu}^{\mathrm{II}}-$ and its ET capability depends on the equilibrium between these two oxidation states by means of the reversible redox reaction $\mathrm{Cu}^{2+}+\mathrm{e}^{-} \rightleftharpoons \mathrm{Cu}^{1+}$, which continuously converts the $\mathrm{Cu}^{\mathrm{II}}$ oxidized state into the $\mathrm{Cu}^{\mathrm{I}}$ reduced state and vice versa. The electronic nature and the peculiar arrangement of the $\mathrm{Cu}^{\mathrm{II}}$ ligands in a distorted trigonal bipyramidal geometry-essentially unchanged in the $\mathrm{Cu}^{\mathrm{I}}$ state ${ }^{[7]}-$ are responsible for its unique properties, such as the intense electron-absorption band at $628 \mathrm{~nm}^{[8]}$ and the unusually high redox equilibrium potential $(+116 \mathrm{mV}$ versus standard calomel electrode [SCE]). A disulfide bridge (between Cys3 and Cys26) is located at one end of the $\beta$-barrel-structure protein and at a distance of $\approx 2.6 \mathrm{~nm}$ from the copper site. ${ }^{[8]}$ It allows the chemisorption of azurin in oriented monolayers onto crystalline gold or other suitably functionalized surfaces. ${ }^{[9,10]}$

Our prototype structure (Fig. 1a) is a planar metal-insulator-metal nanojunction, consisting of two $\mathrm{Cr} / \mathrm{Au}(6 \mathrm{~nm}$ thick Cr layer under a $35 \mathrm{~nm}$ thick Au layer) arrow-shaped metallic electrodes facing each other at the oxide side of a $\mathrm{Si} / \mathrm{SiO}_{2}$ substrate (drain and source electrodes) and connected by the self-assembled protein monolayer. ${ }^{[11]}$ The nanojunction was fabricated by electron-beam lithography, ${ }^{[12]}$ and a silver gate electrode was deposited on the back of the p-doped Si substrate to form an ohmic bond acting as the back gate in a field-effect transistor (FET) configuration. Both the sourcedrain separation and the oxide thickness were $100 \mathrm{~nm}$. Typically, after the fabrication process the contact morphology was inspected by scanning electron microscopy (SEM) and the contacts tested in an open-circuit configuration. On average, about $90 \%$ of the nanojunctions was found to be of good quality with no leakage and with an open-circuit resistance higher than 100-200 G $\Omega$. The protein monolayer was formed in a two-step procedure involving a) the self-assembly of 3mercaptopropyltrimethoxysilane (3-MPTS) and b) the reaction of the free thiol groups of 3-MPTS with the surface disulfide bridge of azurin, which is broken to accomplish covalent bonding to the silane-functionalized $\mathrm{Si} / \mathrm{SiO}_{2}$ substrate. As a single disulfide bond is present in azurin, an oriented monolayer is formed in which all the proteins have the same orientation with respect to the substrate.

Since protein adsorption on surfaces may lead to denaturation, we examined the protein shape and fold pattern after immobilization. A typical non-contact atomic-force microscopy (NC-AFM) image of azurins chemisorbed on $\mathrm{SiO}_{2}$ is displayed in Figure 1b. The surface is totally covered by recognizable granular structures, which are not present on silanized 

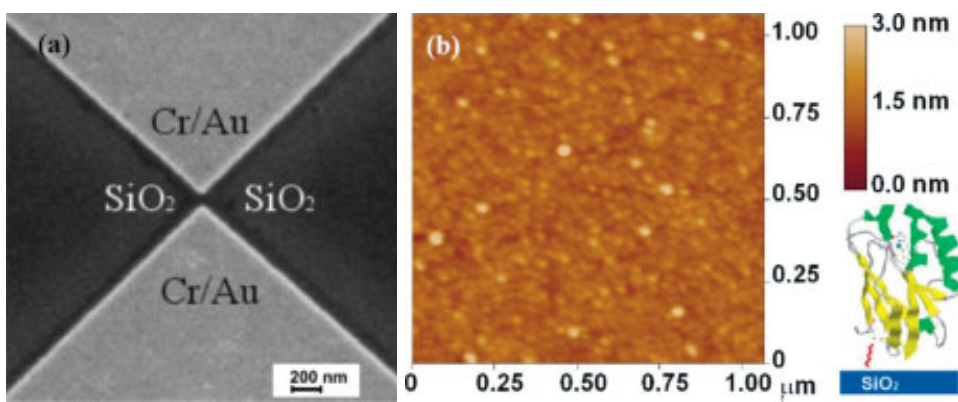

(c)

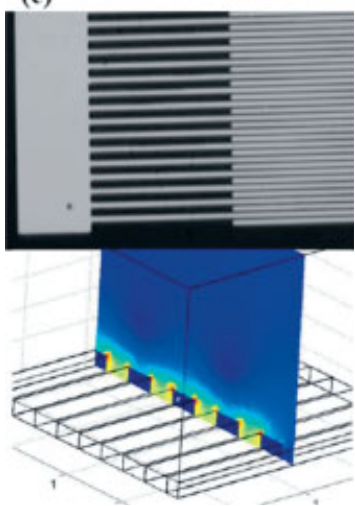

(d)

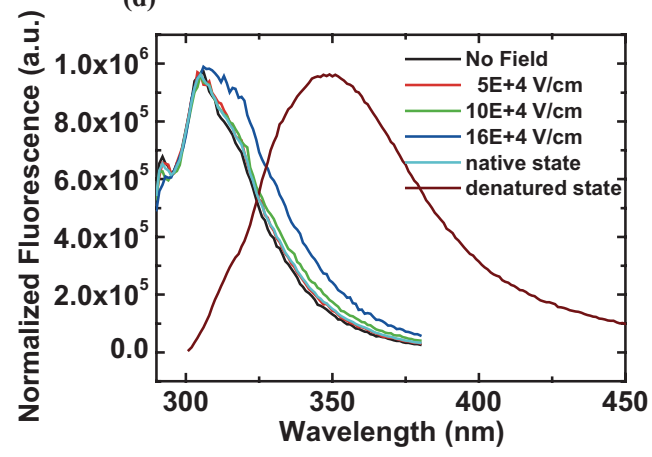

Figure 1. a) High-magnification scanning electron microscopy (SEM) image of two $\mathrm{Cr} / \mathrm{Au}$ nanotips with separation of $100 \mathrm{~nm}$. b) NC-AFM image of an azurin monolayer self-assembled onto functionalized $\mathrm{SiO}_{2}$. Due to the intrinsic loss of resolution introduced by the convolution with the tip geometry, the morphological characterization of these monolayers was performed by a height-distribution analysis, which showed a dominant contribution centered around $4 \mathrm{~nm}$, in general agreement with the size of azurin from X-ray crystallography [13]. c) Top: Photomicrograph of the interdigitated electrodes used in fluorescence experiments to check the integrity of the protein monolayers subjected to an electric field. The structure consists of 500 interdigitated lines of $2 \mu \mathrm{m}$ width and $2 \mu \mathrm{m}$ spacing, resulting in an active area of $2 \mathrm{~mm} \times 2 \mathrm{~mm}$. Bottom: Map of the applied electric field generated with electromagnetic field simulation using finite element methods. The horizontal axes represent the plane of the top panel, while the vertical axis is the distance from the surface of the device. The map is shown in a plane parallel to the vertical distance. The color scale from blue to red corresponds to the visible spectrum, with the red region corresponding to the highest field. d) Fluorescence spectra of native apo-azurin (apo-Az) in solution and on $\mathrm{SiO}_{2}$ without and with an applied electric field. As a reference, the red-shifted emission spectrum of denatured apo-Az in buffer is also reported.

substrates. Since the convolution with probe-tip geometry normally introduces an intrinsic loss of resolution in AFM images, the morphological analysis of the azurin monolayers was performed by a height-distribution analysis, which evidences a dominant contribution (approximately $85 \%$ ) centered around $4 \mathrm{~nm}$. This result is in good agreement with the size of azurin from X-ray crystallography ${ }^{[13]}$ and demonstrates that the chemisorption process does not induce either gross denaturation or conformational transitions, or both, in azurin. As a reference, if the same analysis is performed on monolayers of intentionally denatured azurin $\left(1 \mathrm{~min}\right.$ at $80^{\circ} \mathrm{C}$, before immobilization), the peak due to the intact azurin monomer becomes broader while we observe additional peaks - likely due to the formation of aggregates and to the structural compaction of the proteins-becoming significantly more prominent. No non-specific adsorption is observed in two control experi- ments, one in which proteins are deposited on a gate modified with an amino-functionalized silane, and the other in which a mutant where cysteines Cys 3 and Cys 26 have been replaced with alanines is deposited onto the 3-MPTSfunctionalized gate.

To further support the integrity of azurin in monolayers, we examined their intrinsic fluorescence, since the fluorescence of aromatic residues in proteins is strongly influenced by their microenvironment. A tryptophan residue (Trp48) is responsible for azurin emission, ${ }^{[14,15]}$ and we utilized the apo-form (apo-Az, without the metal atom) for optical investigations, as the fluorescence of the copper form is strongly quenched by the presence of the copper. The fluorescence spectra of apo-Az monolayers and free azurins in buffer (Fig. 1d) are very similar, and only a slight red-shift $(\simeq 2-3 \mathrm{~nm})$ is observed. As a reference, the red-shifted emission spectrum of denatured azurins ${ }^{[16]}$ in buffer is also reported in Figure 1d. The influence of a strong electric field on the protein conformation was studied by means of interdigitated electrodes fabricated using standard photolithographic techniques. The structure consisted of 500 interdigitated gold electrodes with a line-space period of $4 \mu \mathrm{m}$ (Fig. 1c), covering an active area of $2 \mathrm{~mm} \times 2 \mathrm{~mm}$ to which the electric field was applied. The electric-field distribution in such a structure is displayed in Figure 1c. No significant changes in the fluorescence spectra of proteins in electric fields up to $100 \mathrm{kV} \mathrm{cm}^{-1}$ were observed (Fig. 1d). Thus, we conclude that neither the covalent binding to the functionalized $\mathrm{SiO}_{2}$ surface nor the application of strong electric fields induce either gross denaturation or conformational transitions, or both, in azurin. This is very important for possible applications in the field of bioelectronics and biosensors. At field intensities of the order of $100 \mathrm{kV} \mathrm{cm}^{-1}$, a small broadening on the low-energy side of the spectrum is visible. This can be ascribed to a small conformational rearrangement of the protein, which does not imply denaturation or loss of functionality, as compared to the spectrum of denatured azurin.

Current-voltage measurements were carried out on our devices using a semiconductor parameter analyzer (HP Agilent 4155B) in a voltage range between -6 and $6 \mathrm{~V}$ (drainsource voltage) at room temperature and ambient pressure. The gate voltage $\left(V_{\mathrm{g}}\right)$ was changed in order to investigate its influence on the current $\left(I_{\mathrm{ds}}\right)$ between the source $(\mathrm{s})$ and drain (d) electrodes. The current-voltage characteristic $\left(I_{\mathrm{ds}}-V_{\mathrm{ds}}\right)$ of the protein FET (pro-FET) ${ }^{[17]}$ under forward drain-source bias $\left(V_{\mathrm{ds}}\right)$ exhibits a low-current plateau at low field and then rises up to hundreds of picoamperes (Fig. 2). The three-di- 


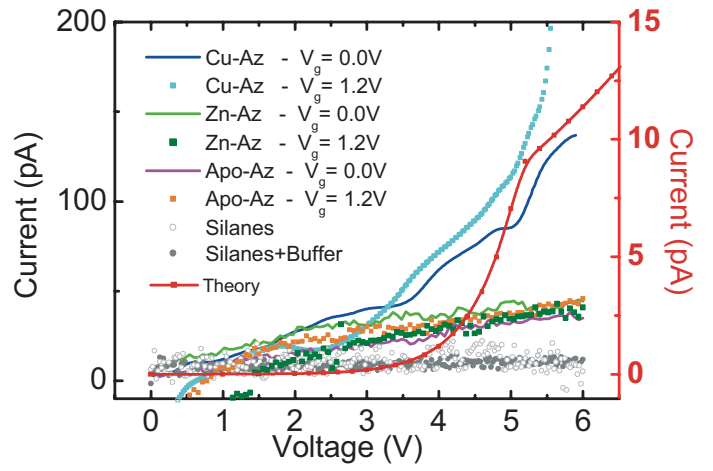

Figure 2. Left scale: Current-voltage characteristics of devices made with azurin variants for two different gate voltages $(0 \mathrm{~V}$ and $1.2 \mathrm{~V})$. As a general feature, devices based on $\mathrm{Zn}-\mathrm{Az}$ or apo-Az exhibited low current (around $50 \mathrm{pA}$ ) with no modulation effect as a function of $V_{\mathrm{g}}$. These results support the interpretation of conduction in proteins and modulation in devices ascribed to the reversible redox reaction $\mathrm{Cu}^{2+}+\mathrm{e}^{-} \rightleftharpoons \mathrm{Cu}^{1+}$, which reconverts continuously the $\mathrm{Cu}^{\prime \prime}$ oxidized state into the $\mathrm{Cu}^{\prime}$ reduced state in adjacent molecules. As a reference, responses are also shown for devices that were either simply silanized, or silanized and incubated in buffer without protein. Right scale: Simulated current-voltage characteristics (red curve) of the pro-FET for a device containing eight protein chains connecting the source and the drain $\left(V_{g}=1 \mathrm{~V}\right.$ was assumed in the calculation).

mensional (3D) plot of the drain-source current $\left(I_{\mathrm{ds}}\right)$ as a function of the drain-source bias $\left(V_{\mathrm{ds}}\right)$ and of the gate bias $\left(V_{\mathrm{g}}\right)$ is displayed in Figure 3. The transfer characteristic exhibits a pronounced resonance centered at $V_{\mathrm{g}}=1.25 \mathrm{~V}$. In this region, the transconductance changes from positive to nega-

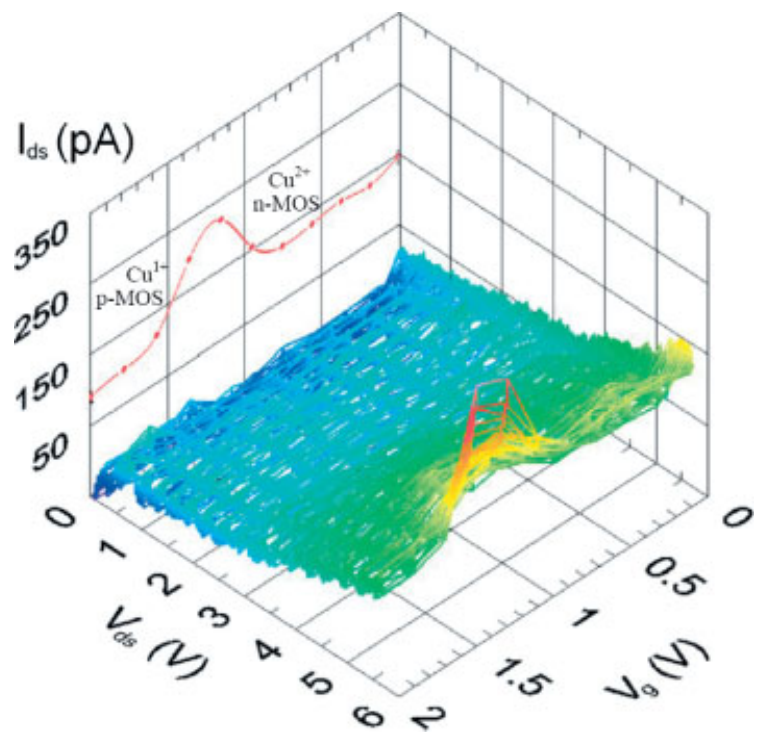

Figure 3. Characteristics of pro-FET: 3D plot of the drain-source current as a function of the drain-source bias $\left(V_{d s}\right)$ and of the gate bias $\left(V_{g}\right)$ measured in the dark and at room temperature. No leakage current was observed to flow between the planar electrodes and the back gate (values as low as few picoamperes and a negligible variation with $V_{\mathrm{g}}$ up to $8 \mathrm{~V}$ ). A pronounced resonance centered at $V_{\mathrm{g}}=1.25 \mathrm{~V}$ is present (see also the transfer characteristic at $V_{\mathrm{ds}}=5.5 \mathrm{~V}$ in the projection). tive values (peak-to-valley ratio of 2 ; full width at half maximum FWHM $=0.3 \mathrm{~V}$ ). This feature gradually disappears after some gate sweeps (Fig. 4). Since the fluorescence experiments in high electric field indicate that azurin still preserves its structure under electric fields as high as $100 \mathrm{kV} \mathrm{cm}^{-1}$, ${ }^{[18]}$ we as-
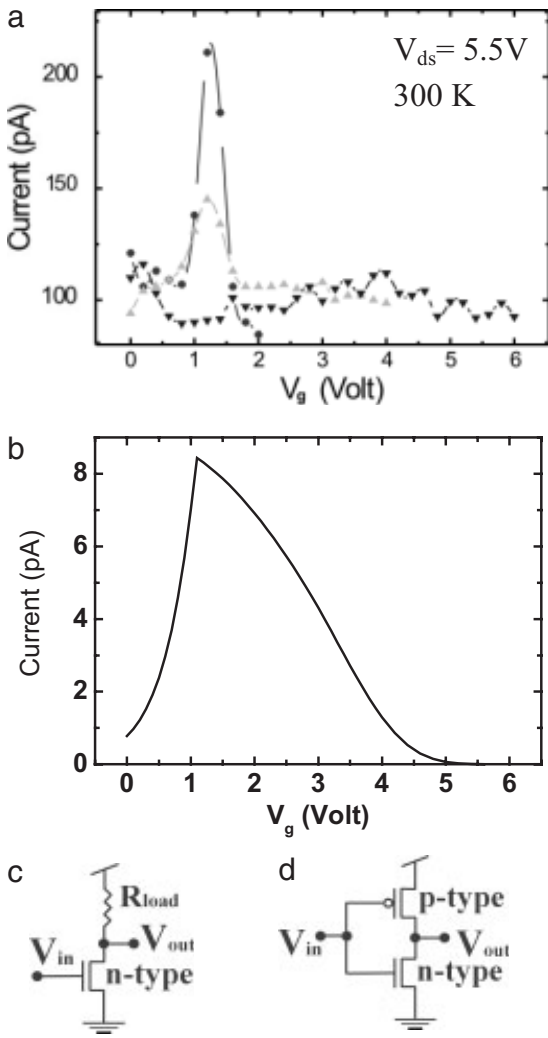

Figure 4. a) Transfer characteristics of the pro-FET: In the resonance region, the transconductance changes from positive to negative values. The peak-to-valley ratio and the FWHM are 2 and $0.3 \mathrm{~V}$, respectively. This feature gradually disappears after some measurement cycles, probably due to metallic contact damage: the curves with dark-grey circles, lightgrey triangles and black inverted triangles were recorded in sequence. b) Simulated transfer characteristic of the pro-FET for a device containing eight protein chains connecting the source and the drain. $V_{\mathrm{ds}}=5 \mathrm{~V}$ was assumed in the calculation. Possible electronic applications of the azurin device, as c) a n-MOS inverter using a resistive load, and d) as a CMOS inverting amplifier. This latter amplifier would have the advantage of consuming power only when switched.

cribe this effect to metallic contact damage induced by the high electric field. ${ }^{[12]}$ Among all the fabricated nanodevices, only a limited group ${ }^{[19]}$ exhibited a clear gate effect over a number of gate sweeps, whereas the others failed during the first few sweeps. The ageing of nanodevices is a general issue, ${ }^{[4,20-22]}$ and electromagnetic field simulations are in progress using finite-element methods in order to further elucidate the failure mechanism.

From an electronic viewpoint, the device switches from behaving as a n-MOSFET before resonance to a p-MOSFET after resonance. This is a key result because it would allow ex- 
ploitation of the advantages of complementary logic, fabricating both p-type and n-type devices on the same chip. For the implementation of an inverter, a unipolar technology requires a load resistance, whereas complementary logic-incorporating both p-type and n-type transistors - would overcome such limitations. This would result in i) a decrease of the logic-gate occupation area (limited to the transistor size scale); ii) a reduction in the fabrication complexity, since in integratedcircuit technology accurate resistors are harder to make than capacitors and transistors; and iii) a decay of power consumption because, as opposed to unipolar inverters that consume power in the low logic state, complementary MOS (CMOS) devices consume power only when switching.

We ascribe these interesting results to the unique transport mechanism of our biomolecular devices. Redox-protein devices are very different from standard inorganic semiconductors and conventional organic devices. Silicon MOSFETs and thin-film transistor (TFTs) are based on a gate field modulating the width and the conductance of a semiconducting channel, whereas a proposed mechanism for carbon-nanotube FETs is Schottky-barrier dominated transport. ${ }^{[23]}$ In ET proteins, the long-range ET, which represents one of the key processes in photosynthesis and respiration, occurs between a donor (D) and an acceptor (A). ${ }^{[8]}$ Two different models for ET in proteins have been proposed, ${ }^{[24]}$ i.e., a superexchange mechanism (consisting of direct quantum tunneling between the donor and acceptor) and a sequential (incoherent) hopping between adjacent sites. The main factors influencing the ET rate ${ }^{[8]}$ are: 1) the distance between the two redox centers (in electron tunneling the ET rate decreases exponentially with distance, whereas in hopping it is inversely related to the distance); 2) the nature of the microenvironment separating the donor and the acceptor (which mediates the virtual state or provides intermediate states, respectively); 3) the reorganization energy $\lambda$, i.e., the energy required for all structural adjustments (in the reactants and in the surrounding molecules) that are needed for the transfer of the electron; ${ }^{[25]}$ and 4) the driving force. In particular, in the case of azurin the essentially unchanged copper-site geometry in the $\mathrm{Cu}^{\mathrm{II}}$ and $\mathrm{Cu}^{\mathrm{I}}$ states minimizes the reorganization energy $\lambda$ and favors fast ET.

The transport of electrons through systems containing redox sites may occur either via physical displacement of the redox molecules, or electron hopping from one reduced molecule to an adjacent oxidized molecule, or both. ${ }^{[26]}$ In our case, the first mechanism can be discarded since the proteins are covalently bound to the substrate and no diffusion of proteins between interdigitated electrodes under high electric fields is observed in AFM images of proteins. Thus, in the pro-FET, electron transport must occur through hopping from one reduced $\left(\mathrm{Cu}^{\mathrm{I}}\right)$ protein to an adjacent oxidized $\left(\mathrm{Cu}^{\mathrm{II}}\right)$ protein (Fig. 5), which behave as a redox pair. For electrons to flow, therefore, both reduced and oxidized azurins must be present, and their relative proportion determines the ET rate. Let us introduce, in analogy to solid-state physics, two functions $f_{\mathrm{Cu}^{2+}}$ and $f_{\mathrm{Cu}^{1+}}=1-f_{\mathrm{Cu}^{2+}}$ that provide the probabilities that a copper site is in the $\mathrm{Cu}^{\mathrm{I}}$ and $\mathrm{Cu}^{\mathrm{II}}$ state, respectively (i.e., the fraction

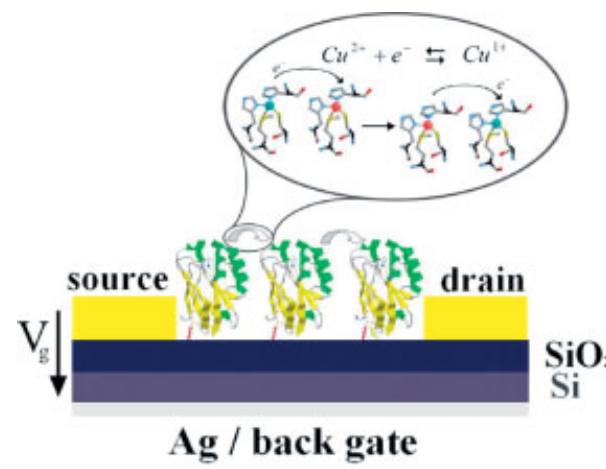

Figure 5. 3D crystal structure of the blue-copper protein azurin containing the central $\mathrm{Cu}$ ion as redox site; cross-section (not to scale) of the pro-FET, and scheme for the transport mechanism. The site geometry of the copper site is a distorted trigonal bipyramid. The disulfide bridge (Cys3-Cys26) opposite the copper atom is exploited to induce chemisorption of azurins on silane-functionalized substrates. The FET consists of a protein monolayer connecting two arrow-shaped $\mathrm{Cr} / \mathrm{Au}$ electrodes on a $\mathrm{SiO}_{2}$ substrate. $\mathrm{An} \mathrm{Ag}$ back-electrode forms an ohmic bond to the silicon that acts as gate. In our model, transport is based on sequential electron hopping between one reduced azurin (blue copper ion in the inset) to an adjacent oxidized one (red ion in the inset). The gate field (vertical) influences the oxidation state of the redox site, originating the resonance.

of reduced and oxidized azurins in the layer). If $W_{\mathrm{im}}$ is the intermolecular transfer rate, the overall ET rate $W_{\mathrm{ET}}$ takes the form

$$
\begin{aligned}
W_{\mathrm{ET}}\left(V_{\mathrm{ds}}, V_{\mathrm{g}}\right)= & W_{\mathrm{im}}\left(V_{\mathrm{ds}}\right) f_{\mathrm{Cu}^{2+}}\left(V_{\mathrm{g}}\right) f_{\mathrm{Cu}^{1+}}\left(V_{\mathrm{g}}\right)= \\
& W_{\mathrm{im}}\left(V_{\mathrm{ds}}\right) f_{\mathrm{Cu}^{2+}}\left(V_{\mathrm{g}}\right)\left[1-f_{\mathrm{Cu}^{2+}}\left(V_{\mathrm{g}}\right)\right]
\end{aligned}
$$

where we assumed that $V_{\mathrm{ds}}$ and $V_{\mathrm{g}}$ influence $W_{\mathrm{im}}$ and $f_{\mathrm{Cu}^{2+}}$, respectively. In other words, we propose that the intermolecular transfer rate $W_{\text {im }}$ depends on the in-plane driving force, which is related to the bias applied between drain and source electrodes (hopping mechanism). On the other hand, the azurin redox state is thought to be regulated by $V_{\mathrm{g}}$ (see below): the higher is $V_{\mathrm{g}}$, the greater is the fraction of reduced azurins. The probability that any two adjacent azurin molecules form a redox pair is $f_{\mathrm{Cu}^{2+}}\left(1-f_{\mathrm{Cu}^{2+}}\right)$. This product is maximal when $f_{\mathrm{Cu}^{2+}}=0.5$, i.e., when the two populations of azurin molecules are present in equal amounts. At a particular value of $V_{\mathrm{g}}$, the fraction of oxidized molecules will equal that of reduced molecules and therefore ET will be maximal. At higher (or conversely, lower) $V_{\mathrm{g}}$ values, the fraction of reduced (or oxidized) molecules will be higher than that of oxidized (or reduced) molecules, resulting in a lower ET rate. It is important to mention that in our model, the change in the protein oxidation state is ascribed to the influence of the applied electric field on the redox energy level of the proteins (since no leakage current is observed).

To verify whether a model based on a hopping mechanism between neighboring proteins is compatible with the experimental findings, we performed numerical simulations of the current flowing in the device as a function of the applied 
potentials. We assumed a square lattice of azurin molecules deposited between the drain and the source, and did not try to introduce into the model adjustable parameters chosen to fit experiments. Instead, we made reasonable a priori assumptions for the various model parameters, based, whenever possible, on independent estimates (such as for the azurin-azurin electron-transfer rate, see computational details in Experimental), and we compared the resulting behaviors with the experimental ones. Only one adjustable parameter was left in the model, the energy difference between the Fermi level of the unbiased electrodes and the redox energy of the protein, and its adjusted value was found, a posteriori, to be very reasonable (see discussion below).

Equation 1, written for a pair of nearest-neighbor proteins, gives the probability per unit time that they exchange an electron. By summing up one such equation for each pair of nearest-neighbor proteins, one obtains a set of equations for the evolution of the probabilities of each molecule to be in the oxidized $\left(\mathrm{Cu}^{2+}\right)$ state as

$$
\begin{aligned}
\frac{\mathrm{d} f^{i}{ }_{\mathrm{Cu}^{2+}}}{\mathrm{d} t}= & \sum_{j} W_{i m}^{i j} f_{\mathrm{Cu}^{2+}}^{j}\left(1-f_{\mathrm{Cu}^{2+}}^{i}\right) \\
& -\sum_{j} W_{i m}^{j i} f_{\mathrm{Cu}^{2+}}^{i}\left(1-f_{\mathrm{Cu}^{j}}^{2+}\right)
\end{aligned}
$$

which is written for a given protein $i$ in the device geometry, and the $j$-sums run over all the nearest neighbors of $i$. We numerically solved the set of evolution Equations 2, finding the probability $f_{\mathrm{Cu}^{2+}}$ for each protein at each instant. Details of the computational scheme are reported in the Experimental section. From the knowledge of the $f_{\mathrm{Cu}^{2+}}$ probabilities, the current intensity at a given instant is finally obtained by calculating the total rate of ET from an electrode (source or drain) to the proteins. In fact, this flux represents the total electronic current entering the protein layer from that electrode. Since the computational burden rapidly grows as a function of the number of proteins, we performed most of the calculations with only a few (eight) parallel chains of proteins connecting the drain and the source, and we verified what happens on increasing the number of chains.

The resulting current-potential characteristics obtained from the hopping-based model are depicted, together with the corresponding experimental results, in Figure 2 and Figure 4. The basic features of the experimental curves are indeed consistent with the results of our model: we refer, in particular, to the resonance of the drain-source current as a function of the gate potential (Fig. 4b) and the threshold-like behavior of the current as a function of the drain-source potential (Fig. 2). The presence of these features is particularly noteworthy. However, as expected for a model with non-optimized parameters, discrepancies with the precise experimental behaviors are present (e.g., the calculated $I_{\mathrm{ds}}-V_{\mathrm{g}}$ peak is broader and more asymmetric than the experimental one, but note that the broadest part of the experimental peak may be hidden by the background current). The most evident discrepancy is the difference between the numerical and the experimental magni- tude of the current. We remark, however, that the calculations were performed for only eight protein chains. When more chains to bridge drain and source electrodes are considered, the resonance and threshold-like features of the current are conserved, but the intensity increases. ${ }^{[27]}$ Other parameters, such as the azurin-azurin ET rate, the azurin reorganization energy, or the detailed geometry of the electrodes during the device operation can affect the current magnitude: no attempt was made to optimize the value of these parameters. As a final comment on the suitability of the hopping-based model to reproduce clear experimental features, we emphasize that in this numerical model only one adjustable parameter is present, i.e., the energy difference between the Fermi levels of the unbiased electrodes and the redox energy of the protein (see computational details). We chose the value of this parameter $(0.45 \mathrm{eV})$ to reproduce in the calculation the same resonance peak position as in the experiment. Such a value compares rather well with $0.4 \mathrm{eV}$, i.e., the difference obtained from the work function of polycrystalline gold (the metal constituting the bulk of the electrodes), which is $5.3 \mathrm{eV},{ }^{[28]}$ and the absolute redox potential of azurin in aqueous solution ( $0.3 \mathrm{~V}$ versus normal hydrogen electrode [NHE, where NHE versus vacuum is $4.6 \mathrm{eV}])$. Although the environmental conditions in the pro-FET are obviously not the same as for azurin in solution and gold in vacuum, such an agreement corroborates the reliability of the model. The proposed model is also consistent with the interpretation of the redox peak in cyclic voltammetry curves and in electrochemical scanning tunneling microscopy experiments ${ }^{[10,29]}$ performed on azurins chemisorbed onto $\mathrm{Au}(111)$ substrates.

The key role of the copper atom in ET is further supported by a comparison with the current-voltage curves measured in devices implemented with two azurin variants: the first with the $\mathrm{Cu}$ atom replaced by a $\mathrm{Zn}$ atom $(\mathrm{Zn}-\mathrm{Az})$, and the second without a metal atom (apo-Az). In Figure 2, the $I-V$ characteristics for two different gate voltages $(0$ and $1.2 \mathrm{~V}$, the latter corresponding to the resonance in the transfer characteristics of the $\mathrm{Cu}$ azurin devices shown in Fig. 4) are displayed for each protein device. The currents flowing through devices based on the two variants are significantly lower, and no modulation is observed between -6 and $6 \mathrm{~V}$. We ascribe the low conductivity of these devices to the absence of a metal atom capable to mediate ET, since apo-Az contains no metal atom, while $\mathrm{Zn}$ has only one viable oxidation number $(+2)$. As a reference, the $I-V$ characteristics of devices silanized, or silanized and incubated in buffer without protein, are also reported in the same figure. In these cases the currents are lower and comparable with the noise level of empty devices.

In conclusion, we report a pro-FET exploiting the redox and self-assembly properties of the metallo-protein azurin and propose a working mechanism in terms of intermolecular hopping, based on the continuous reconversion of the copperoxidized state $\mathrm{Cu}^{\mathrm{II}}$ into the reduced state $\mathrm{Cu}^{\mathrm{I}}$. The novelty of both the active material and the transport mechanism makes such a device very interesting for molecular-electronics applications. 


\section{Experimental}

Materials: Wild-type azurin obtained from $P$. aeruginosa was purified by chromatography using carboxymethyl (CM) Sepharose Fast Flow (FF) and diethylaminoethyl (DEAE) Sepharose, essentially as previously described $[30,31]$. The product was characterized by sodium dodecylsulfate poly(acrylamide) gel electrophoresis (SDSPAGE) and UV-vis spectroscopy, and stored at $-20^{\circ} \mathrm{C}$ in $20 \mathrm{mM} 4-(2-$ hydroxyethyl)-1-piperazineethanesulfonic acid (HEPES) $\mathrm{pH} 7$ buffer. Apo-azurin and $\mathrm{Zn}$-azurin were prepared essentially as described in the literature [31-33] and stored as the wild-type protein.

Procedure: The technique for protein immobilization is based upon the self-assembly of 3-mercaptopropyltrimethoxysilane (3-MPTS). The $\mathrm{SiO}_{2}$ substrates were washed in sequence with trichloroethylene, acetone, methanol, and 2-propanol, incubated with $\mathrm{H}_{2} \mathrm{SO}_{4} / \mathrm{H}_{2} \mathrm{O}_{2}$ (3:1) overnight, and then washed with abundant water. In order to obtain ordered monolayers whilst preventing multilayer formation, the substrate was incubated in a very diluted solution $(2 \%)$ of 3 -MPTS for 2 min. After washing in absolute ethanol and drying the sample under a nitrogen stream, azurin was chemisorbed onto the silane layer by depositing a drop of the protein solution $\left(0.6 \mu \mathrm{g} \mathrm{ml}^{-1}\right)$ and incubating for $5 \mathrm{~min}$ at room temperature while shaking. Protein immobilization takes place via the reaction of the free thiol groups of 3-MPTS with the surface disulfide bridge of azurin, giving rise to substrate-overlayer disulfide bonds. The samples were imaged by non-contact atomic force microscopy (NC-AFM).

Lithography: Interdigitated electrodes with a line-space period of $4 \mu \mathrm{m}$ were defined in $\mathrm{AZ} 5214$ resist on $\mathrm{Si} / \mathrm{SiO}_{2}$ substrates using standard photolithographic techniques. Planar nanojunctions [12] were fabricated on thermally oxidized silicon wafers using a combination of photolithography and electron-beam lithography (EBL, Leica Lion LV1 system). Photolithography followed by lift-off was used to define the Ag $50 \mathrm{~nm}$ thick gate electrode on the back of the $\mathrm{Si} / \mathrm{SiO}_{2}$ substrate and the $\mathrm{Cr} / \mathrm{Au}$ contact pads on $\mathrm{SiO}_{2}$. Subsequently, an EBL process was carried out using the Leica Lion LV1 system (having a $5 \mathrm{~nm}$ point-size electron beam) to expose the poly(methyl methacrylate) (PMMA) electronic resist (molecular weight of $950 \mathrm{~kg} \mathrm{~mol}^{-1}$ ) spin-coated on the $\mathrm{Si} / \mathrm{SiO}_{2}$ substrate at $7000 \mathrm{rpm}$ for $40 \mathrm{~s}$ (final thickness of $240 \mathrm{~nm}$ ). Typical exposure parameters were: accelerating voltage of $5 \mathrm{keV}$, beam current in the range $10-20 \mathrm{pA}$, and writing dose of about $50 \mu \mathrm{C} \mathrm{cm}^{-2}$. After electron beam exposure, samples were developed in a mixture of one part of methyl isobutyl ketone (MIBK) and three parts of isopropanol (IPA) for $100 \mathrm{~s}$. Finally, metal electrodes were obtained by electron beam metal evaporation $(\mathrm{Cr} / \mathrm{Au}$, layer thicknesses $6 \mathrm{~nm}$ and $30 \mathrm{~nm}$ ) followed by lift-off in an acetone bath for more than $10 \mathrm{~min}$ at moderate temperatures $\left(35-40^{\circ} \mathrm{C}\right)$. All the fabricated nanojunctions were inspected by plan-view scanning electron microscopy in order to establish the success of the whole process and to estimate the separation between the nanotips. Typically, about $90 \%$ of nanojunctions were of good quality with no leakage and an open-circuit resistance higher than 100-200 G $\Omega$.

Instrumentation: NC-AFM images were taken with a BioScope microscope (Digital Instruments) equipped with a $90 \mu \mathrm{m}$ scanner at a scanning rate of $1 \mathrm{~Hz}$. Probes were $\mathrm{Si}_{3} \mathrm{~N}_{4}$ tips with a $10 \mathrm{~nm}$ nominal curvature radius. Fluorescence emission spectra were recorded in photon-counting mode using a $450 \mathrm{~W}$ xenon lamp for excitation and double monochromators both in excitation and emission. To monitor azurin luminescence from protein films, the exciting radiation was focused on the protein film, which was held with its normal at $30^{\circ}$ with respect to the direction of the exciting beam, while the front-surface emission was collected at $90^{\circ}$ from the excitation. All the emission spectra on $\mathrm{SiO}_{2}$ were recorded at room temperature $\left(20^{\circ} \mathrm{C}\right)$, atmospheric pressure, and $54 \%$ humidity. The excitation wavelength was $280 \mathrm{~nm}$ ( $2 \mathrm{~nm}$ bandwidth). Electrical characterization of nanodevices was performed by current-voltage measurements using a semiconductor parameter analyzer (HP Agilent 4155B). Typically, the integration time was $20 \mathrm{~ms}$, while the wait time between successive sweeps was around $1 \mathrm{~s}[19]$.
Computational Details: To quantify the intermolecular transfer rate $\left(W_{\mathrm{im}}\right)$ term for the solution of Equation 2, we exploited Marcus's theory [34], the standard theory for ET between molecules. The ingredients of Marcus's expression for the ET rate are the transfer integrals between the molecules, the reorganization energy, and the driving force for the redox process. We estimated the transfer integrals between two azurin molecules from reasonable values for the azurin electron self-exchange reaction rate constant $\left(k_{\mathrm{ESE}}\right)$ in azurin dimer constructs $k_{\mathrm{ESE}}=5 \times 10^{5} \mathrm{~s}^{-1}$ consistent with the lower-limit experimental estimate [35]. The reorganization energy was taken equal to $0.7 \mathrm{eV}$, similar to what has been experimentally found for the azurin electron self-exchange reaction $(0.6-0.8 \mathrm{eV})$ [36]. Finally, we assumed that the driving force for the hopping process is the electrostatic-potential difference between the two molecules exchanging the electron due to the applied source-drain bias. In fact, the redox energy level $\varepsilon_{1}$ of each molecule changes as a function of the electrostatic potential $V_{\mathrm{i}}$ felt by the molecule as $\varepsilon_{\mathrm{i}}=\varepsilon_{0}-e V_{\mathrm{i}}$, where $\varepsilon_{0}$ is the zero-field redox energy. The source-drain bias creates a potential difference (and consequently an energy difference) between two molecules placed along the source-to-drain direction. In addition, $V_{\mathrm{g}}$ modulates the redox behavior of the protein: if $V_{\mathrm{g}}$ is increased, the energy of the redox level decreases and the protein is more prone to be reduced. To obtain a reliable estimate of $V_{i}$ for all the molecules in the monolayer, we computed the electrostatic potential distribution in the real device by means of finite-element methods. For the molecules in direct contact with the source or the drain, we assumed that the electrode-molecule electron-transfer rate was much larger than the intermolecular one; i.e., we considered these interface proteins to be in thermodynamic equilibrium with the electrodes. Thus, their redox state is regulated by the difference $\varepsilon_{\mathrm{i}}-\varepsilon_{\mathrm{f}}=\varepsilon_{0}-e V_{\mathrm{i}}-\varepsilon_{\mathrm{f}}$ between the redox level of the protein and the electrochemical potential $\varepsilon_{\mathrm{f}}$ of the attached electrode The difference $\varepsilon_{0}-\varepsilon_{\mathrm{f}}$ remained as the only adjustable parameter in our model.

Received: April 26, 2004 Final version: December 9, 2004

[1] A. Aviram, M. A. Ratner, Chem. Phys. Lett. 1974, 29, 277.

[2] G. Maruccio, P. Visconti, V. Arima, S. D'Amico, A. Biasco, E. D'Amone, R. Cingolani, R. Rinaldi, Nano Lett. 2003, 3, 479.

[3] J. Appenzeller, J. Knoch, V. Derycke, R. Martel, S. Wind, P. Avouris, Phys. Rev. Lett. 2002, 89, 126801

[4] S. J. Tans, A. R. M. Verschueren, C. Dekker, Nature 1998, 393, 49.

[5] J. Lefebvre, J. F. Lynch, M. Llaguno, M. Radosavljevic, A. T. Johnson, Appl. Phys. Lett. 1999, 75, 3014.

[6] E. Vijgenboom, J. E. Busch, G. W. Canters, Microbiology 1997, 143 , 2853.

[7] G. Battistuzzi, M. Borsari, L. Loschi, F. Righi, M. Sola, J. Am. Chem. Soc. 1999, 121, 501.

[8] O. Farver, I. Pecht, J. Am. Chem. Soc. 1992, 114, 5764.

[9] Q. Chi, J. Zhang, J. U. Nielsen, E. P. Friis, I. Chorkendorff, G. W. Canters, J. E. T. Andersen, J. Ulstrup, J. Am. Chem. Soc. 2000, 122, 4047.

[10] A. Alessandrini, M. Gerunda, G. W. Canters, M. P. Verbeet, P. Facci, Chem. Phys. Lett. 2003, 376, 625.

[11] Self-assembly is a very interesting tool for the implementation of molecular devices and can be defined as the recognition and the subsequent self-organization of different elements, without any external source of information about the structure to be formed, as the total free energy of the system is minimized to result in a more stable state.

[12] G. Maruccio, P. Visconti, S. D'Amico, P. Calogiuri, E. D'Amone, R. Cingolani, R. Rinaldi, Microelectron. Eng. 2003, 67-68, 838.

[13] H. Nar, A. Messerschmidt, R. Huber, M. van de Kamp, G. W. Canters, J. Mol. Biol. 1991, 221, 765.

[14] S. J. Kroes, G. W. Canters, G. Gilardi, A. Van Hoek, A. J. Visser, Biophys. J. 1998, 75, 2441 
[15] A. Finazzi-Agrò, G. Rotilio, L. Avigliano, P. Guerrieri, V. Boffi, B. Mondovì, Biochemistry 1970, 9, 2009.

[16] The unfolding process results in a large solvent exposure of hydrophobic residues that were shielded in the core of the protein in its native state.

[17] We call our protein device a field-effect transistor since we exploit a field effect to modulate the resistance of the protein monolayer and change the $I-V$ characteristics of the device.

[18] The field in the actual device is at least one order of magnitude larger and we cannot investigate directly these fields in fluorescence experiments (since it would be necessary to apply hundreds of volts). However, the magnitude of azurin inner fields (as calculated from the molecular electrostatic potential) is larger than $10^{7} \mathrm{~V} \mathrm{~m}^{-1}$ over nearly the whole protein volume, and larger than $10^{8} \mathrm{~V} \mathrm{~m}^{-1}$ in about $40 \%$ of the space. Thus, it seems reasonable that the fold pattern of the biased proteins is also unperturbed by the electric field in the device, which is lower.

[19] More than 10 devices fabricated in different times and measured in different conditions exhibited ET. The shape of the characteristics remained the same when the integrations and the delay times were changed.

[20] J. Park, A. N. Pasupathy, J. I. Goldsmith, C. Chang, Y. Yaish, J. R. Petta, M. Rinkoski, J. P. Sethna, H. D. Abruna, P. L. McEuen, C. Ralph, Nature 2002, 417, 722.

[21] a) J. Lee, G. Lientschnig, F. Wiertz, M. Struijk, R. A. J. Janssen, R. Egberink, D. N. Reinhoudt, P. Hadley, C. Dekker, Nano Lett. 2003, 3, 113. b) C. R. Kagan, A. Afzali, R. Martel, L. M. Gignac, P. M. Solomon, A. G. Schrott, B. Ek, Nano Lett. 2003, 3, 119.

[22] R. M. Metzger, B. Chen, U. Hopfner, M. V. Lakshmikantham, D. Vuillaume, T. Kawai, X. Wu, H. Tachibana, T. V. Hughes, H. Sakurai, J. W. Baldwin, C. Hosch, M. P. Cava, L. Brehmer, G. J. Ashwell, J. Am. Chem. Soc. 1997, 119, 10455.

[23] S. Heinze, J. Tersoff, R. Martel, V. Derycke, J. Appenzeller, Ph. Avouris, Phys. Rev. Lett. 2002, 89, 106801.

[24] O. Kuhn, V. Rupasov, S. Mukamel, J. Chem. Phys. 1996, 104, 5821.

[25] A. D. McNaught, A. Wilkinson, IUPAC Compendium Of Chemical Terminology, 2nd ed., IUPAC, Research Triangle Park, New York 1997.

[26] D. N. Blauch, J. M. Saveant, J. Am. Chem. Soc. 1992, 114, 3323.

[27] For example, with 50 chains the maximum current is $35 \mathrm{pA}$. We remark that the number of active chains actually present in the experimental device is difficult to determine, but 50 chains (corresponding to approx. 2500 proteins, which is already computationally very demanding) is actually a conservative estimate.

[28] H. B. Michaelson, J. Appl. Phys. 1977, 48, 4729.

[29] P. Facci, D. Alliata, S. Cannistraro, Ultramicroscopy 2001, 89, 291.

[30] G. W. Canters, FEBS Lett. 1987, 212, 168.

[31] M. van de Kamp, F. C. Hali, N. Rosato, A. Finazzi-Agro, G. W. Canters, Biochim. Biophys. Acta 1990, 1019, 283.

[32] H. Nar, R. Huber, A. Messerschmidt, A. C. Filipou, M. Barth, M. Jaquinod, M. van de Kamp, G. W. Canters, Eur. J. Biochem. 1992, 205, 1123.

[33] H. Nar, A. Messerschmidt, R. Huber, M. van de Kamp, G. W. Canters, FEBS Lett. 1992, 306, 119.

[34] R. A. Marcus, N. Sutin, Biochim. Biophys. Acta 1985, 811, 265.

[35] I. M. C. van Amsterdam, M. Ubbink, A. Messerschmidt, A. Merli, D. Cavazzini, G. L. Rossi, G. W. Canters, Nat. Struct. Biol. 2002, 9, 48.

[36] a) A. J. Di Bilio, M. G. Hill, N. Bonander, B. G. Karlsson, R. M. Villahermosa, B. G. Malmström, J. R. Winkler, H. B. Gray, J. Am. Chem. Soc. 1997, 119, 9921. b) H. B. Gray, B. G. Malmström, R. J. P. Williams, JBIC, J. Biol. Inorg. Chem. 2000, 5, 551.

\section{Novel Light-Emitting Diodes Using Organic Electroluminescent Nanocapsules**}

\author{
By Jae-Seok Heo, No-Hyung Park, Jee-Hyun Ryu, and \\ Kyung-Do Suh*
}

Electroluminescence (EL) has been of interest for several decades because of its potential applications in fields such as telecommunications and information display. Light-emitting diodes (LEDs) utilizing $\mathrm{p}-\mathrm{n}$ junctions of various inorganic semiconductors have dominated the scene during the last twenty years. However, the deposition of inorganic semiconducting materials as thin films on the electrode surface necessitates relatively expensive and inconvenient techniques, such as the high-vacuum vapor deposition process, which are not applicable to large-area device fabrication. ${ }^{[1]}$

First observed from anthracene in $1963,{ }^{[2]}$ organic EL initially had low efficiencies and lifetimes of devices compared to inorganic EL. Tang and Van Slyke ${ }^{[3]}$ and Saito et al. ${ }^{[4,5]}$ made breakthrough developments, however, using an organic fluorescent dye, which led to a new generation of LEDs in the late 1980s. Since then, both academia and industry have seen an explosion of activity in organic EL. ${ }^{[6]}$ Generally, organic LEDs are classified as one of two types, depending on the nature of the active emitting materials: they either consist of small conjugated molecules or of a conjugated polymer. ${ }^{[1,7]}$ Small-molecular EL materials show low driving voltage and high quantum and luminescence efficiency, ${ }^{[1,8,9]}$ but their excitation quenching, charge entrapping, and catalytic degradation prevent application in LEDs. ${ }^{[9]}$ While polymer-type EL materials have several merits, including tailorability and easy fabrication at low cost ${ }^{[10,11]}$ they need improved quantum efficiency and operational lifetime. ${ }^{[12]}$ Recently, research has benefited from combining the advantages of small-molecular and polymeric EL materials. In this study, our approach is to en-

[*] Dr. K.-D. Suh, J.-H. Ryu

Division of Chemical Engineering

College of Engineering

Hanyang University

Seoul 133-791 (Korea)

E-mail: kdsuh@hanyang.ac.kr

I.-S. Heo

Industrial Materials R\&D

LG Chem., Ltd.

Cheong-ju Plant

150, Songjeong-dong, Hungduk-gu, Cheongju, 361-721 (Korea)

Dr. N.-H. Park

Industrial Materials R\&D

LG Chem, Ltd.

104-1, Moonji-dong, Yuseong-gu, Daejeon 305-380 (Korea)

[**] This study was supported by a grant of the Korea Health $21 \mathrm{R \& D}$ Project, Ministry of Health \& Welfare, Republic of Korea (03-P)1PG1-CH14-0001). 